\title{
Desktop Mapping
}

Desktop Publishing in der Kartographie, ein Anwendungsbeispiel

\section{Einleitung}

Die Kartenherstellung war schon immer ein Gebiet, das nicht ausschließlich Kartographen vorbehalten war. Die im Bereich der Automation entstandenen Werkzeuge verstärken die Tendenz zu einer VergröBerung des Kreises der nichtprofessionellen Kartenhersteller weiter: So ist z. B. schon seit längerem eine graphische und kartographische Visualisierung von räumlichen Daten beispielsweise direkt aus Statistik-Softwarepaketen möglich (und aus Geographischen Informationssystemen geradezu selbstverständlich). Eine kritische Beurteilung der verfügbaren Programme zeigt aber, da $\beta$ diese noch einige Mängel aufweisen (vgl. z. B. CARTER 1987, BAKER et al. 1985 und GREEN et al. 1985).

Neuere technische Entwicklungen haben andererseits die Möglichkeit geschaffen, direkt am Arbeitsplatz druckreife Dokumente zu erstellen. Die entsprechenden Voraussetzungen dazu wurden in den letzten Jahren durch die Einführung entsprechender Hard- und Software gemacht: Personal Computer und Laserdrucker stehen heute in vielen Büros. Es ist leicht vorauszusehen, dass dieses "Desktop Publishing" in naher Zukunft auch Auswirkungen auf die Menge kartographischer Erzeugnisse haben wird: genauso wie heute jeder Bericht mit ein paar Business-Graphiken angereichert wird, wird es beim Vorhandensein entsprechender Software auch möglich sein, gleiches mit Karten zu tun. «The skills of cartography, like so many other professions, are becoming „key-board skills” - possessed by anyone and everyone» (JUPE 1987, 341).

\section{Desktop Publishing}

Der Bereich des Desktop Publishing ist heute einer der rasch expandierenden Märkte im EDV-Bereich. Vor allem das Druckgewerbe sieht sich dabei einem neuerlichen Innovationsschub gegenüber. Die Entwicklung einer Palette von Ausgabemedien (vom vergleichsweise preisgünstigen Laserdrucker bis zum teuren Lichtsatzgerät), welche verschiedenartige Qualitätsbedürfnisse abdecken können, hat hier - in Verbindung mit Software auf kleinen Rechnern - neue Möglichkeiten eröffnet. Zentral ist dabei, daß«(...) einfach, kurzfristig und kostengün- stig direkt am Arbeitsplatz und ohne Hilfe von Spezialisten" Druckerzeugnisse hergestellt werden können (Apple-Werbung, Hervorhebung durch den Verfasser).

Die Qualität der mit diesen neuen Mitteln erzielten Resultate ist aber unter Fachleuten nicht unbestritten: Schon die ersten Erfahrungen haben eine gewisse Ernüchterung gebracht, indem sie gezeigt haben, $\mathrm{da} ß$ die mit diesen neuen Mitteln hergestellten Druckerzeugnisse auf den ersten Blick wohl professionell aussehen, daß aber häufig graphische, typographische (und sicher bald auch kartographische) Analphabeten am Werk sind.

Eine eingehende Auseinandersetzung der Fachleute aus den betroffenen Disziplinen (eingeschlossen der Kartographie) mit den Möglichkeiten des Desktop Publishing scheint notwendig1. Dazu gehört zunächst die Evaluation der Möglichkeiten, die sich durch den Einsatz der neuen Informatikmittel ergeben ${ }^{2}$. Zu den weiteren Aufgaben sind sicher auch die Entwicklung von kartographisch robusten Methoden, das Bereitstellen von geeigneten Werkzeugen und entsprechende Ausbildungsanstrengungen zu zählen. Zweck der Bemühungen wäre es, sicherzustellen, daß die einfach zu handhabenden Mittel des Desktop Publishing zur Erstellung von kartographisch ansprechenden Karten verwendet werden. Die von typographischen Laien produzierten Druckerzeugnisse lassen häufig nicht nur den Fachmann erschaudern; gleiches im Bereich der Kartographie zu verhindern, scheint eine lohnenswerte Aufgabe zu sein. Dies kann mit verschiedenen Mitteln erreicht werden, im Idealfall könnten solche Anstrengungen in die Entwicklung eines Expertensystems münden (Ansätze dazu z. B. bei MULLER 1986).

Adrian Herzog, dipl. Geograph, Geographisches Institut, Universität Zürich-Irchel, Winterthurerstraße 190, 8057 Zürich 


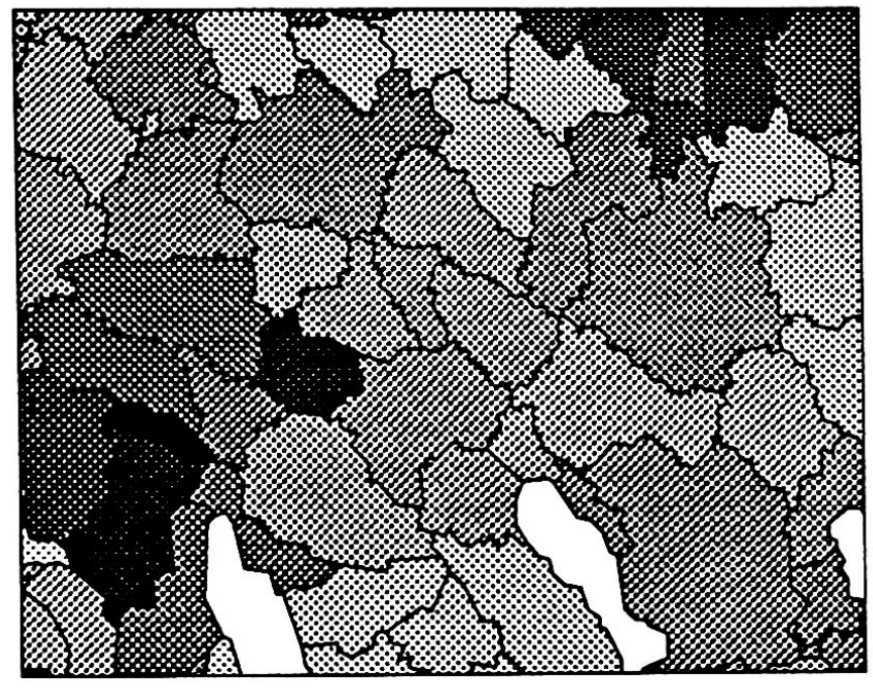

Abb. 1 a) lineare Schwärzungskurve,

\section{Das Programm PS-Copam}

Ein erster Versuch, hier die Hard- und SoftwareMittel des Desktop Publishing in der Kartographie einzusetzen, war die Implementation eines einfachen Choroplethenkartenprogramms, das im folgenden näher beschrieben werden soll.

Das Programm PS-Copam ${ }^{3}$ basiert auf dem von TOBLER (1973) vorgeschlagenen und später von BRASSEL und UTANO (1979) verfeinerten Konzept für die Herstellung klassenloser Choroplethenkarten: Die einzelnen Gebietseinheiten erhalten einen ihrem Variablenwert entsprechenden Grauwert, die Einzelwerte werden dabei vorgängig nicht klassiert ${ }^{4}$. Dieses einfache Konzept führt in der Regel zu einem differenzierten Bild einer räumlichen Verteilung und schränkt die Möglichkeiten für unzweckmäßige oder geradezu falsche Symbolisierungen stark ein.

In bezug auf die konkrete Symbolisierung wurden hier die für Pen-Plotter konzipierten Methoden durch Halbton-Verfahren ersetzt, die speziell bei den im Bereich des Desktop Publishing heute verwendeten Laserdruckern und Lichtsatzgeräten üblich sind: Diese Rastergeräte emulieren verschiedene Grauwerte durch den Aufbau eines Druckrasters. Dieser wiederum wird aus kleinen Punkten (üblicherweise bei Laserdruckern mindestens 300 dots per inch, bzw. ca. 120 Linien je $\mathrm{cm}$, bei Lichtsatzgeräten ab ca. 500 Linien je $\mathrm{cm}$ ) aufgebaut.

Der Schwärzungsverlauf kann im Programm mittels verschiedener Parameter modifiziert werden. Zum einen geschieht dies durch die Festsetzung von Schwellenwerten, über die hinaus die kontinuierliche Veränderung des Grauwerts nicht weiter fortgesetzt wird; der Einfluß von Ausreißerwerten kann dadurch verringert werden. Zum anderen kann die

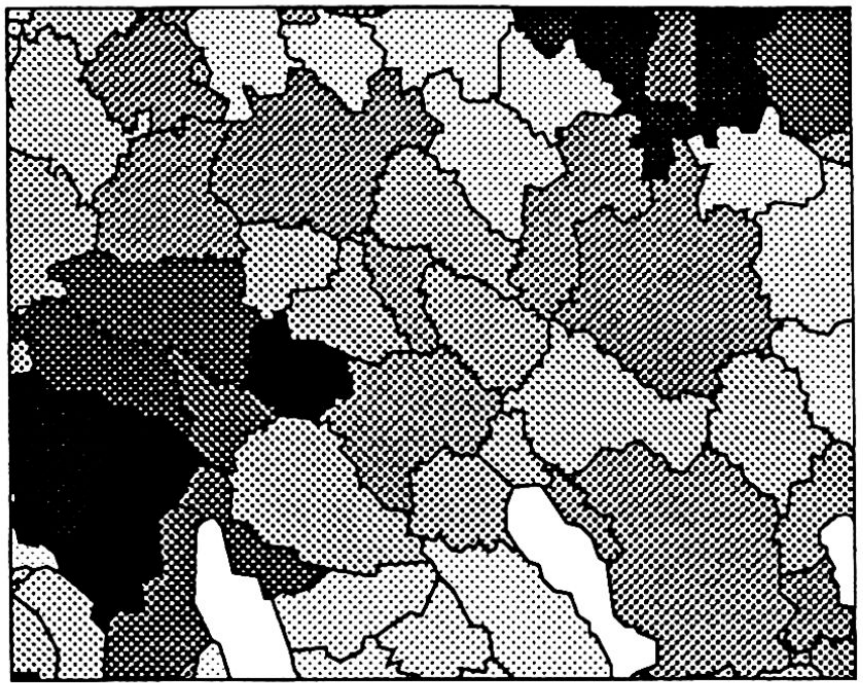

b) logistisch verformte Schwärzungskurve

Schwärzungskurve exponentiell oder - in Erweiterung des bisherigen Konzepts - logistisch verformt werden. Eine logistische Verformung führt zu einem größeren Kontrast in der Karte: derjenige Bereich des Variablenumfangs, in dem üblicherweise die meisten Datenwerte liegen, kann damit differenzierter dargestellt werden, der Kontrast der Gesamtkarte wird erhöht (vgl. Abb. 1).

Im weiteren erlaubt das Programm eine Beschriftung der Karte mit einer Vielzahl von Schriftarten in kontinuierlich festlegbaren Schriftgrößen.

Zur Beschriftung der einzelnen Gebietseinheiten konnte ebenfalls eine Eigenschaft von Rastergeräten ausgenutzt werden: Graphische Informationen, welche zu einem späteren Zeitpunkt gezeichnet werden, überschreiben das schon Gezeichnete. So werden zunächst die einzelnen Gebietseinheiten mit einem ihrem Variablenwert entsprechenden Grauwert gefüllt, anschließend kann der Gebietsname darübergeschrieben werden. Dabei wurde der Versuch unternommen, die Schrift nicht wie bisher üblich mit einer rechteckigen Fläche zu unterlegen, sondern mit einem "Halo» zu umgeben (vgl. Abb. 3 und 4).

Die Umrisse der einzelnen Gebietseinheiten (inklusive allfälliger En- bzw. Exklaven) werden dem Programm in einer sequentiellen Datei als einzelne geschlossene Polygone übergeben. Dabei wird zum einen mit schweizerischen Gemeinden und darauf aufbauenden Regionalisierungen gearbeitet (vgl. HERZOG et al. 1983), zum andern wurde eine Schnittstelle zum Geographischen Informationssystem ARC/INFO geschaffen. Mit diesem GIS können zunächst beliebige Umrisse digitalisiert werden; anschließend erfolgt eine Umwandlung in eine topologisch saubere Struktur und das Herausschreiben der gebildeten Polygone. 


\section{Ein Anwendungsbeispiel: Karten in Zeitungen}

«Without a doubt, maps which appear in the mass media are seen by more people today than any other type of map. (. . .) Researchers in related fields such as graphic design, journalism, and cartography have paid little attention to journalistic graphics, however.» (GILMARTIN 1985,1; vgl. auch McDERMOTT 1987, 32f.). Dem Desinteresse der Kartographie steht ein zunehmender Bedarf nach Graphik jeder Art gegenüber, der dazu geführt hat, daß auch Zeitungen heute eine immer größere Zahl von graphischen Elementen - und damit auch Karten - enthalten. Dabei stehen Karten im Vordergrund, die es dem Leser ermöglichen sollen, den Ort eines aktuellen Ereignisses zu lokalisieren. Aber auch thematische Karten finden sich in Zeitungen: So wird beispielsweise die Analyse von Abstimmungsergebnissen häufig durch Karten unterstützt, die meist unter starkem Zeitdruck entstehen. Die kartographische Qualität variiert dabei aber beträchtlich, wobei das Schwergewicht eher bei mißverständlichen, ungeeignet symbolisierten Karten liegt. Dabei wird gerade einer elementaren Anforderung an eine Karte in einer Zeitung kaum Rechnung getragen: ein rasches Erfassen der räumlichen Verteilung ist häufig nicht möglich, und die Karte kann damit ihre Funktion als Erklärungshilfe kaum erfüllen.

Als Beispiele für Abstimmungskarten in Zeitungen seien zwei aktuelle herausgegriffen. Im einen Fall («Neue Zürcher Zeitung», 7.12.87, S. 18) findet man eine Karte zur Rothenthurm-Initiative (Abb. 2).

Die üblichen gleichabständigen Klassen erfüllen ihren $Z$ weck recht gut, unbefriedigend ist allerdings deren Umsetzung in Grauwerte: Die beiden mittleren Klassen unterscheiden sich nur durch die Signaturmuster, ihre Grauwerte unterscheiden sich aber kaum, die Klasse mit den kleineren Werten wirkt sogar eher noch etwas heller als diejenige mit den gröBeren Werten.

Ein Vergleich mit einer klassenlosen Choroplethenkarte (Abb. 3) zeigt, daß gerade auch die Klassenbildung ihre Tücken haben kann: Während die Kantone Zürich und Bern (58,7 bzw. $60,3 \%$ Zustimmung, Differenz: $1,6 \%$ ) sehr ähnliche Werte aufweisen, aber in verschiedene Klassen zu liegen kommen, wird der mehr als doppelt so große Unterschied zwischen den Kantonen Freiburg und Waadt (59,9 bzw. $56,0 \%$ Ja-Stimmen, Differenz: 3,9\%) durch die Klassenbildung verdeckt.

Als zweites Beispiel sei eine Karte zur Abstimmung über die Kranken- und Mutterschaftsversicherung erwähnt («Tages-Anzeiger», 7.12.87, S. 9). Wiederum finden sich dort vier gleichabständige Klassen, diese wurden aber so ungünstig gewählt, daß beinahe die ganze Fläche der Schweiz in dieselbe Klasse zu liegen kommt (prozentuale Flächenanteile der Klassen: $7-1-91-1)^{5}$. Erst bei näherem
Hinsehen entdeckt man einzelne von der dominanten Klasse abweichende Kantone.

In einem Test wurde nun untersucht, ob es mit den heute verfügbaren Mitteln des Desktop Publishing möglich ist, innert nützlicher Frist und mit geringem Aufwand Karten von ansprechender Qualität - und eventuell auch höherem Detaillierungsgrad - herzustellen. In Zusammenarbeit mit einer Photoagentur (Keystone) wurde die Brauchbarkeit der Konzepte getestet. So wurden an zwei Abstimmungswochenenden Karten hergestellt: Karten der Schweiz nach Kantonen und (unveröffentlicht) eine Karte des Kantons Zürich mit Gemeindeergebnissen einer kantonalen Vorlage. Beim ersten Versuch wurden die Karten auf den üblichen Vertriebskanälen (Funk) den Abonnenten der Agentur übermittelt; trotz der zum Teil sehr störenden Moiré-Effekte wurden diese von mehreren Zeitungen übernommen (29.9.86). Bei einem zweiten Versuch wurde die Originalvorlage einer Zeitung überbracht, die dabei erzielte Druckqualität kann als sehr gut bezeichnet werden («NZZ», 8.12.86, S. 18). Da das Layout der Karte - und damit beinahe sämtliche $\mathrm{Pa}$ rameter - schon vor dem Abstimmungswochenende festgelegt werden konnte, blieb einzig die Erfassung der 26 Kantons- bzw. 188 Gemeinde- und Stadtkreis-Ergebnisse und das Ausdrucken der Karte. ${ }^{6}$ Dadurch daß mit automatischen Mitteln auch relativ große Datenmengen in kurzer Zeit visualisiert werden können, eröffnen sich für die Detailanalyse von räumlichen Verteilungen Möglichkeiten, die mit herkömmlichen Mitteln innert nützlicher Frist kaum zu erreichen wären (vgl. Abb. 4).

\section{Schluß}

Hard- und Softwaremittel des Desktop Publishing eignen sich für kartographische Belange gut, dies haben die Versuche mit dem Choroplethenkartenprogramm PS-Copam gezeigt. Das Programm erweist sich vor allem durch die einfache Bedienung und die in der Regel schon durch die Voreinstellungswerte erreichten befriedigenden Resultate als praktisches und robustes Instrument zur Visualisierung statistischer Oberflächen. Mit geringem Aufwand können verschiedene Varianten verglichen werden, die graphische Qualität der LaserdruckerAusgabe dürfte für die meisten Ansprüche genügen. Wünschenswert wäre eine vorgängie Visualisierung am Bildschirm, eine interaktiv-graphische Festlegung der Parameter, eventuell eine farbgetrennte Ausgabe und eine Erweiterung durch andere Kartiermethoden.

Das Fehlen von sauberen Umrißdateien bzw. die geringe Verbreitung von Geographischen Informationssystemen im Kleincomputerbereich werden wohl zunächst eine rasche Verbreitung des «Desk- 


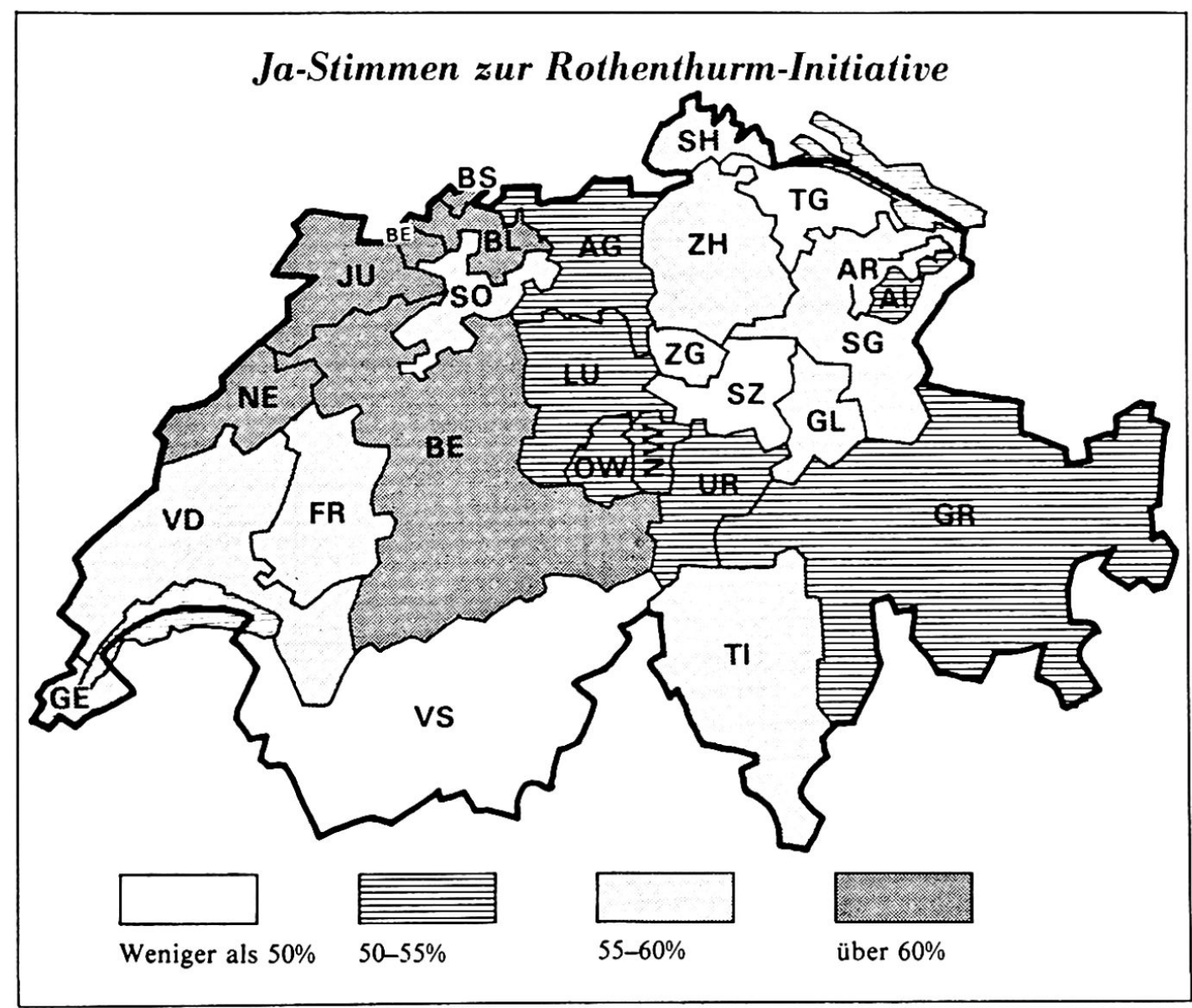

Abb. 2 Abstimmungskarte "NZZ", 7. 12.87

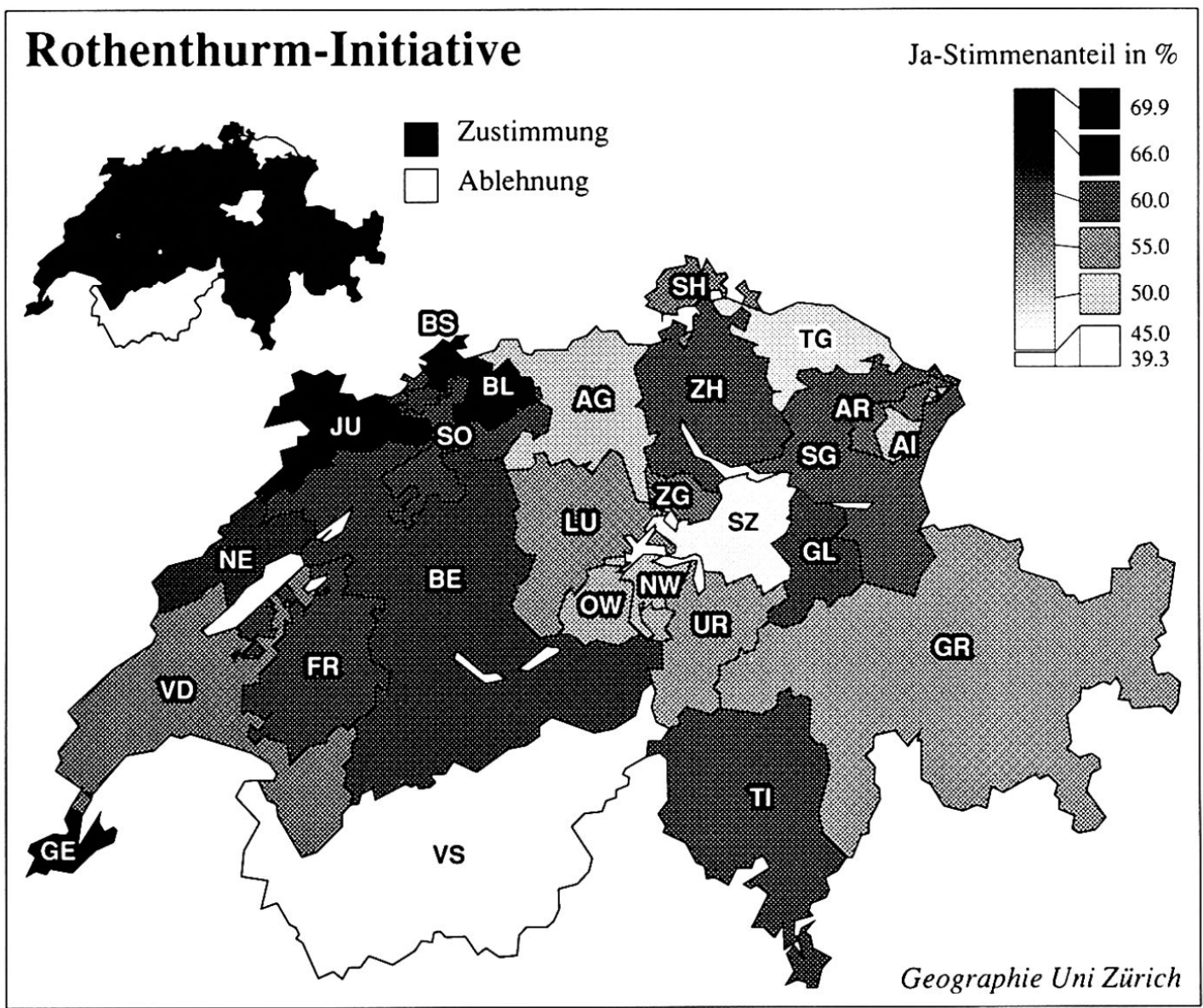

Abb. 3 Abstimmungskarte mit PS-Copam 


\section{Stimmrechtsalter 18}

Ja-Stimmenanteil in \%

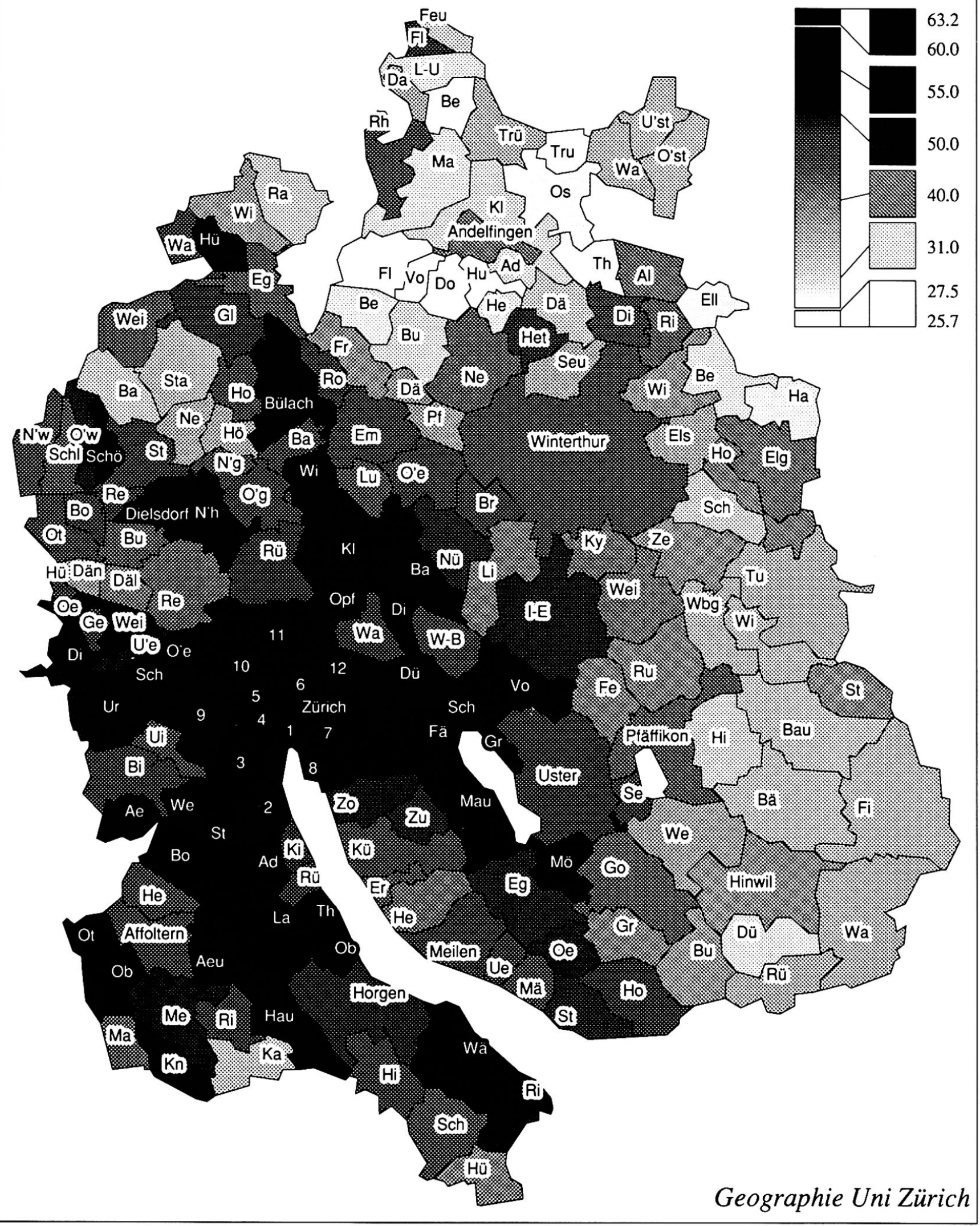

Abb. 4 Abstimmungskarte Kanton Zürich, Versuch mit abgekürzten Gemeindenamen 
top Mapping» verhindern. Bis zu dem Zeitpunkt, wo dieser Mangel behoben sein wird, müssen aber Softwarepakete entwickelt sein, die die Herstellung von kartographisch einwandfreien Karten sicherstellen können.

\section{Anmerkungen}

1 Die Diskussion dazu findet im typographischen Bereich schon intensiv statt (vgl. z. B. FRITZSCHE 1987).

${ }^{2}$ vgl. dazu z. B. die "Software Reviews" im American Cartographer oder die "Reviews of Geographic Software" im Professional Geographer. In beiden werden häufig auch Programme aus dem PC-Bereich besprochen. Darunter finden sich bisher kaum Kartenprogramme, die als positiv bewertet werden konnten. Dies führt dazu, daß heute behelfsmäßig vielfach Programme für die Kartenherstellung verwendet werden, welche nicht dafür konzipiert wurden und die sich deshalb für diesen Zweck schlecht eignen.

${ }^{3}$ Computertechnisches: Das Programm ist in StandardFORTRAN 77 geschrieben. Die Implementation des Programms erfolgte auf einem Apple Macintosh, wobei dessen hohe Interaktionsfähigkeit und graphische Möglichkeiten nicht ausgenutzt wurden. Der Programmablauf ist mehr oder weniger batch-orientiert, d. h. nach Eingabe der für das Programm notwendigen Informationen, die vorgängig in verschiedenen Dateien abgelegt worden sind, werden die gesamten Berechnungen durchgeführt, ohne daß eine interaktive Modifikation von Parametern möglich wäre. Die graphische Information wird dem Ausgabemedium mittels einer Seitenbeschreibungssprache übermittelt, wobei hier mit der weitverbreiteten «PostScript -Sprache (ADOBE 1985) gearbeitet wird. Diese Programmiersprache hat sich in diesem Zusammenhang sehr bewährt, bietet sie doch große Flexibilität in bezug auf die graphische Ausgestaltung einer Druckseite. Bei ausreichender Kenntnis der PostScript-Sprache kann der Benutzer beispielsweise auf relativ einfache Art noch mit spezifischen Halbtonverfahren experimentieren. Das Programm PS-Copam erzeugt somit eine Datei (ein PostScript-Programm), die anschließend auf dem Ausgabegerät interpretiert und ausgegeben wird. Insofern genügt PS-Copam gewissen Eigenschaften des Desktop Publishing nicht: das zentrale Prinzip WYSIWYG ("what you see is what you get") wird (noch) nicht erfüllt. Die großen Datenmengen bei der räumlichen Datenverarbeitung stellen dabei für kleine Rechner ein beachtliches Hindernis dar.

Die Zeit für die Programmausführung beträgt auf einem Macintosh SE für die zweiteilige Kantonskarte (Abb. 3) ca. 1:15 min., die Verarbeitung auf einem LaserWriter Plus ca. 2:25 min. (Gemeindekarte Kt. Zürich, 188 Gebiete, 6753 Polygonpunkte, keine Gemeindenamen: 2:47, bzw. 3:20 min.).

${ }^{4}$ Auf die umfangreiche Diskussion, die das Konzept klassenloser Karten ausgelöst hat und die die Kartographenwelt in zwei Lager gespalten hat, soll hier nicht weiter eingegangen werden, man vergleiche dazu die Literaturhinweise.
${ }^{5}$ Gemäss einer mündlichen Mitteilung lag in diesem Fall die Ursache bei einem voreiligen Redaktionsentscheid.

6 Das Konzept der klassenlosen Karten enweist sich dabei auch insofern als vorteilhaft, als keine Verzögerungen durch das Experimentieren mit verschiedenen Klassenzahlen bzw. -grenzen entstehen.

\section{Literatur}

ADOBE (1985): PostScript Language Reference Manual, Reading MA. etc.: Addison-Wesley

BAKER, A. M., R. FALUDI und D. R. GREEN (1985): "An Evaluation of SAS/GRAPH Software for Computer Cartography". In: Professional Geographer, 37 (2), 204-214

BRASSEL, K. E. und J. J. UTANO (1979): “Design Strategies for Continuous-tone Area Mapping." In: The American Cartographer, 6 (1), 39-50.

CARTER, J.R. (1987): "SAS/GRAPH" und "SPSS GRAPHICS" (Software Reviews). In: The American Cartographer, 14 (2), 164-175.

COULSON, M. R. C. (1987): "In the Matter of Class Intervals for Choropleth Maps: With Particular Reference to the Work of George F. Jenks". In: Cartographica, 24 (2), 16-39.

DOBSON, M. (1973): "Choropleth Maps Without Class Intervals?: A Comment". In: Geographical Analysis, 5 (4), 358-360.

FRITZSCHE, J. (1987): «DTP - oder (erst) Durch Typographie Perfekt». In: Druckindustrie, 19, 6.10.87, 99.

GILMARTIN, P. (1985): “The Design of Journalistic Maps: Purposes, Parameters and Prospects". In: Cartographica, 22 (4), 1-18.

GREEN, D. R., A. M. OMMER, A. L. DEETH, A. NUZZO and R. FALUDI (1985): "SAS/GRAPH for Cartography: Map Projections and Labelled Choropleth Maps". In: Cartographica, $22(2), 63-78$.

HERZOG, A., K. BRASSEL, M. BOPP und G. DORIGO (1983): "Die automatische Generierung von Grenzliniendateien der Schweiz". In: Geographica Helvetica, 38 (3), $112-120$

JUPE, D. (1987): “The New Technology: Will Cartography Need the Cartographer?». In: The Canadian Surveyor, 41 (3), 341-346.

MCDERMOTT, P. D. (1987): “Journalistic Cartography, An Essay". In: Tech. Papers 1987 ASPRS-ACSM Annual Convention, Vol. 4: Cartography, 32-41.

MULLER, J. C. (1986): "Construction des cartes générales à petite échelle à l'aide d'un système expert". In: Bulletin du Comité Français de Cartographie, fascicule no 109-110, bulletin no 3-4, 89-94 (Séminaire Euro-Carto V).

TOBLER, W. R. (1973): “Choropleth Maps Without Class Intervals?». In: Geographical Analysis, 5 (3), 262-265. 